\title{
Patología Informacional
}

\section{Informational Pathology}

\author{
Sebastián Loma-Osorio ${ }^{1}$ \\ Nadine Schramm ${ }^{2}$ \\ Guy Santibáñez-H ${ }^{3}$
}

\begin{abstract}
Resumen
Esta presentación está dedicada a mostrar aspectos de la patología informacional debidos al efecto que el exceso de información tiene sobre el organismo.

Si un organismo - humano o animal - es obligado a asimilar una cantidad de información que satisface una fuerte motivación, sin disponer del tiempo necesario para procesarla, sufre un estado tensional (estrés), el cual produce una patología que Chananashwili denominó "neurosis informacional". Esta alteración tiene su base fisiopatológica en el Síndrome General de Adaptación, descrito por Selye (1950).

El progreso tecnológico, las exigencias laborales y las modernas condiciones de vida, han favorecido la falta de actividad, la necesidad de tomar importantes decisiones en períodos cortos de tiempo, lo cual conduce a situaciones de estrés de larga duración. Esto afecta a determinados sistemas funcionales, a la aparición de los así llamados síndromes psicosomáticos, intensas reacciones emocionales y síntomas como angustia, ansiedad y depresiones además de otros.

Dada la importancia que ésta patología adquiere en la actualidad, hemos querido dar un cuadro sintético, tanto en el aspecto clínico como en el experimental y, al mismo tiempo, señalar su relación directa con la dinámica de los acontecimientos económico-sociales.
\end{abstract}

Palabras claves: Información, neurosis, patología

\begin{abstract}
This paper reports some aspects of the informational pathology that is due to effect which excess of information has on living organisms.

If a human being or animal is compelled to assimilate a large amount of information under a strong motivational pressure, without having the time necessary to process
\end{abstract}

${ }^{1}$ Ayudante. Departamento de Psicología. Universidad de Chile, Chile.

${ }^{2}$ Ayudante. Departamento de Psicología. Universidad de Chile, Chile.

${ }^{3}$ Profesor Titular. Departamento de Psicología. Universidad de Chile, gsantiba@uchile.cl 
the information, the organism will experience a tensional state (stress). This will be responsible for a pathological condition, called "informational stress" by Chananaswili. The physiopathological basis of this alteration lies in the General Adaptation Syndrome described by Selye (1950).

Technological progress, excessive demands of work, the "modern way of life" that stimulates sedentary life styles, and the requeriments to make short time decisions, all configure a stressing environment. This affects various functional systems, thus generating profound emotional alterations, psychosomatic syndromes, symptoms such as anxiety and depression.

As this pathology is becoming ever more pervasive, we wish to synthetically emphasize some of its clinical and experimental aspects, besides pointing out its increasing importance in social economic life.

Key words: Stress, information, pathology

\section{Concepciones Básicas}

Es tal vez necesario entregar una somera información de los fundamentos básicos de las contribuciones de Selye (1950), el Síndrome General de Adaptación (GAS), puesto que el estrés es el centro de la patología informacional.

La palabra "stress", traducida al lenguaje corriente, significa "estado tensional" producido por una serie inespecífica de agentes que operan sobre el organismo, el cual tiene un correlato tanto corporal como subjetivo. La gama de estímulos que son capaces de desencadenar este síndrome va desde situaciones psicológicas hasta estímulos de carácter fisico-químicos, a condición de que sus parámetros no sobrepasen en intensidad, duración o frecuencia a las capacidades de dichos organismos de asimilarlos, sin poner el peligro su equilibrio adaptativo (Selye, 1956).

Selye describe el así llamado "Systemic Stress" (1950, p.286y sig.) como la respuesta de las suprarrenales a la acción de la hormona adrenocorticotrofica (ACTH) secretada por el lóbulo anterior de la hipófisis, una vez que ésta glándula ha sido activada por información proveniente del hipotálamo, a través del sistema hipotálamo-hipofisiario.
La respuesta del organismo a la activación de las suprarrenales es lo que Selye llamo "Síndrome General de Adaptación".

Todo este proceso estaría desencadenado por estímulos que inespecíficamente son capaces de participar en la generación de diversos tipos de patologías, pero que, al mismo tiempo, participan en la eclosión del Síndrome General de Adaptación, previas a la reacción hipotálamo-hipofisissuprarrenal.

Adaptación es la habilidad que tiene, por ejemplo, el organismo de enfrentar al medio externo en sus variaciones, es decir, a las condiciones dinámicas del ambiente. De una manera análoga, el organismo enfrenta a las variaciones del medio interno que pueden inducirse por acción de agentes patógenos, por cambios metabólicos, por acción de agentes térmicos $u$ otros que son productores de tensiones subjetivoconductuales o subjetivo-viscerales. Un fenómeno semejante tiene lugar si existe una infección bacteriana o viral, un fuerte dolor o una prolongada activación emocional. La reacción defensiva del organismo para enfrenta los agentes o elementos patógenos, es un intento para neutralizarlos y retrotraer el ambiente interno a una condición de variabilidad controlada. 
Las propiedades defensivas del organismo se alteran si sus disponibilidades de ACTH disminuyen o aumentan en relación a sus rangos óptimos, sucede algo similar si las hormonas secretadas por las suprarrenales (corticoides, catecolaminas) se encuentran en proporciones inadecuadas.

Nikolov (1956), estudiando los efectos de la hormona adrenocortical sobre los procesos de condicionamiento clásico, encontró que perros parcialmente adrenolectomizados no estuvieron en condiciones de formar reflejos condicionados estables. Antiguos trabajos realizados en el laboratorio de Pavlov (1934), sugieren que las condiciones del entrenamiento de sus perros pueden provocar una patología de la actividad nerviosa superior, debilitando los procesos excitatorios y acortando el tiempo de activación, poniendo en evidencia que las estructuras neurales envueltas en estos procesos perdían la propiedad de integrarse de una manera funcional adecuada.

Los experimentos de Nikolov (1956) mostraron que el aumento de corticoides y de hormona adrenocorticotrofica (ACTH) más allá de los niveles fisiológicamente normales, fenómeno que Selye llamo "enfermedad de hiperadaptación", producía una multitud de efectos, algunos directos y otros a través de las interferencias metabólicas que actuaban sobre la homeostasis. Este aumento de las mencionadas hormonas interfiere con las funciones neuroendocrinas $\mathrm{y}$, en consecuencia, sobre la actividad integrativa del sistema neuroendocrino (Santibáñez-H, 1987, pp. 43-73, 30-38).

Además, Nikolov (1956) mostró una influencia activante directa de esas hormonas sobre la actividad de los interoceptores y sobre neuronas de la medula espinal. Hechos estos que indicarían un efecto modulador sobre la reactividad del sistema neuroendocrino (Telcharov y Nikolov, 1963).

Sudakov $(1980,1986)$ ha llevado a cabo experimentos en ratas y conejos en los cuales los animales fueron estimulados eléctricamente en el núcleo ventro medial del tálamo y, como consecuencia, los animales experimentaron fuertes crisis emocionales acompañadas de diversas reacciones motoras, aumento de la actividad cardiaca y de la presión arterial. Estas manifestaciones se hicieron crónicas con todas las manifestaciones de la activación hormonal de estrés.

Airapetyants y Vein (1982) y Airapetyants (1986) se han preocupado de la etiología de las neurosis, tanto desde un punto de vista clínico como experimental. Han comunicado que el estado tensional de origen emocional puede conducir a diversas situaciones patológicas. Principalmente a tres:

1. A síntomas neurosomáticos (psicosomáticos), tales como la hipertensión, diabetes melitus, asma bronquial, tireotoxicosis.

2. Bajo la influencia de una tensión emocional relativamente intensa y crónica, había síntomas neuróticos de diversa índole. Esto sucede siempre que el estrés emocional esté acompañado de conflictos que afecten el nivel subjetivo.

3. Hay una tercera modalidad de afección: la aparición de síntomas neurovegetativos, los cuales son fácilmente reversibles.

Natelson (1983), presentó una revisión de algunos experimentos llevados a cabo en monos, que darían cuenta del carácter psicosomático de la aparición de úlceras a nivel del estómago distal y del duodeno. Un grupo de investigadores del Instituto de Investigación Militar Walter Reed, reportaron que monos trabajando en evitar shocks - pero sin que los controles impidieran la entrega del shock - se enfermaron y murieron al cabo de un mes. El examen post-mortem reveló la presencia de úlceras pépticas en todos los monos que participaron del experimento. 
En el presente trabajo queremos presentar los resultados de investigaciones que ponen en evidencia alteraciones del equilibrio adaptativo de los seres vivos - hombres y animales - debido a las condiciones especificas en las cuales se produce la interacción individuo-ambiente.

El progreso científico-tecnológico, la pérdida del contacto directo con la naturaleza, la excesiva permanencia en áreas urbanizadas, la falta de actividad corporal, el excesivo requerimiento a tomar decisiones para satisfacer nuestras necesidades imperiosas y, por último, la sensación crónica de falta de tiempo, facilitan considerablemente los episodios de aumento tensional. Se generan permanentemente reacciona emocionales repetitivas de elevado tono simpático que afectan el sistema circulatorio y el sistema muscular, produciendo angustia, agresión y frustración.

Diversos estímulos físicos, tales como el ruido, el smog, el estar sometido a temperaturas extremas pueden determinar respuestas inespecíficas del organismo. Sin embargo, es cada vez más evidente que factores de tipo psicológico y psicosocial originen este tipo de respuestas. Estos factores estresantes pueden generar, con mucha frecuencia, reacciones que son experimentadas como desagradables o intolerables y pueden, incluso, llegar a ser destructivas y determinar alteraciones funcionales y estructurales del organismo (Biondi y Picardi, 1996).

\section{Neurosis Informacional: Datos Clinicos y Experimentales}

Expondremos el concepto de neurosis informacional que señala una forma de patología de las funciones integrativas del sistema neuroendocrino, la cual ha alcanzado un importante aumento en la sociedad contemporánea.
Reproducimos aquí las contribuciones de M.M. Chananashwili y sus colaboradores, que han suministrado una serie de datos empíricos, los cuales nos permiten dar una idea de la problemática que tratamos (Chananashwili, 1984).

\section{Neurosis Informacional: Experimentos en Perros}

Los animales fueron condicionados a tres reflejos pavlovianos alimenticios. El entrenamiento se llevó a cabo en una sala en el centro de la cual había colocada una alfombra que constituía el lugar desde donde el animal y el investigador daban curso al proceso de condicionamiento.

Tres fuentes para contener alimento fueron ubicadas frente al lugar desde donde partía el animal. Estas fuentes estaban separadas entre sí por una distancia de un par de metros. Estas fuentes eran designadas como 1, 2 y 3.

Los perros aprendieron a buscar comida en cada una de las fuentes señaladas por un estimulo especifico: la fuente 1 era señalada por la combinación de una luz y un timbre; la fuente 2 por un tono de $600 \mathrm{Hertz}$ y la fuente 3 por ruido de agua corriente. Los animales aprendieron a buscar y encontrar la comida directamente en la fuente señalada por el estimulo. Los perros estaban fuertemente deprivados de alimento.

El sonido de un metrónomo señalaba que no había comida en ninguna de las fuentes. Los animales aprendieron a inhibir la reacción de búsqueda cuando este estímulo auditivo aparecía.

Una vez que el condicionamiento estaba fuertemente establecido se inicio la segunda fase del experimento. 
En ésta fase del experimento se activaron simultáneamente dos de los estímulos que indicaban comida en dos de las fuentes. Esta combinación produjo una reorganización de la conducta puesta en acción, lo cual indicaba una integración funcional estructuralmente diferente en el sistema nervioso. Los animales comían sin problemas de las dos bandejas.

En la tercera fase del experimento, se administraron a los animales los tres estímulos condicionados en conjunto, simultáneamente. Los resultados fueron indicadores de un notable trastorno de la actividad del sistema neuroendocrino. Los animales se negaron a comer, aparecieron episodios de micción espontanea, cambios respiratorios, desarrollo de tics nerviosos en las extremidades, aumento de las conductas agresivas, llanto, intranquilidad y taquicardia, fragilidad ósea, alopecia.

Las respuestas a la activación unitaria de los reflejos condicionados se hicieron inestables y los animales cometieron una serie de errores que no hacían antes de la tercera fase.

Se trataba de un trastorno generalizado que se mantenía desde 7 días hasta 4 meses después de concluidos los experimentos. Los perros no restablecieron la actividad normal en el ejercicio de reflejos condicionados adquiridos, lo cual indicó el carácter crónico del trastorno.

La velocidad de la instauración del proceso de neurotización depende del nivel motivacional y se puede discriminar o evitar bajando la intensidad de la tensión motivacional. Sin embargo, hay que tener presente que la génesis está ligada al factor tiempo. Hay tres factores que determinan la génesis de la neurotización: un déficit temporal, un nivel motivacional intenso y una excesiva cantidad de información que es necesaria de asimilar.
Los experimentos de control fueron diseñados de manera tal que la combinación de los tres factores señalados fueron optimizados, es decir, se utilizo una cantidad de información que podía ser fácilmente asimilada en el tiempo utilizado y el nivel motivacional no alcanzo limites excesivos.

El déficit de información significativa puede incluir un déficit de intercambio informacional interindividual. En experimentos realizados en monos, bajo condiciones de aislamiento social, han demostrado que esas condiciones generan sintamos de neurosis informacional.

\section{Neurosis Informacional en el Ser Humano}

Se han examinado una serie de personas, las cuales se encontraban en condiciones laborales que implicaban tareas que exigían una gran cantidad de información. Esta información debía ser asimilada, procesada e incorporada a un flujo conceptual indispensable para la continuidad normal del trabajo. Las condiciones patológicas en los diferentes ambientes laborales resultaban de las exigencias que, además de procesar gran cantidad de información, exigían tomar decisiones rápidamente para el desarrollo de la actividad laboral. Estas demandas requerían rápidos cambios de atención, la cual debía pasar de un asunto a otro. Todo esto debía ser resuelto en un lapso sumamente corto y bajo la enorme presión de la responsabilidad que esto significaba, además del sentimiento - aspecto motivacional - con que había que llevar a cabo la tarea con éxito.

Tales circunstancias generan una situación de estrés que conforman un conjunto de reacciones depresivas, angustiosas o agresivas, alteraciones cardio-respiratorias, digestivas, alteraciones del ciclo sueño-vi- 
gilia, pérdida del apetito, inhabilidad para ejecutar las tareas requeridas, situaciones conflictivas que aumentaban la intensidad del estrés.

En esta constelación de sintamos encontramos los mismos comportamientos que hemos examinado en la neurosis informacional animal. A saber: excesiva información, tiempo de elaboración insuficiente y una demanda motivacional intensa. Este mismo síndrome puede encontrarse en el medio escolar, especialmente en los periodos de prueba. Los síntomas que se presentan en los casos clínicos correspondientes son irritabilidad, impulsividad, ansiedad, alteración de la capacidad de concentración, sueño, jaqueca, fatigabilidad, apatía, alteración de la digestión, perdida de peso, sudoración y hasta alopecia.

Es interesante mencionar aquí que si el sujeto es alejado de su contexto laboral o escolar, los sintamos desaparecen de manera relativamente rápida. Sin embargo, al volverlo a sus condiciones habituales, la sintomatología se organiza nuevamente.

\section{Discusión}

El concepto de neurosis informacional designa una forma especial de patología de las funciones integrativas del sistema neuroendocrino (Konorski, 1967). Este sistema trabaja en tres condiciones cuando se desencadena este síndrome. Ellas son, según Chananashwili (1984): necesidad de incorporación y procesamiento de una cantidad desproporcionada de información; déficit temporal para desarrollar esa actividad y un nivel motivacional muy elevado envuelto en el procesamiento.

El déficit informacional no sólo puede deberse lisa y llanamente a la falta de información, sino también al suministro de información, aunque muy intenso, no sig- nificativa. Desde un punto de vista funcional, el exceso de información significativa y el déficit de información significativa en un contexto informacional excesivo tiene como resultado final la privación de la información necesaria para mantener las posibilidades adaptativas del sistema neuroendocrino.

Entendemos por información, las señales que los individuos pueden asimilar, proveniente de los hechos, sucesos o estímulos de una manera directa o a través de sistemas comunicacionales.

Examinando el problema desde un punto de vista etiopatogénico, la patología informacional es una alteración de las funciones integrativas del sistema neuroendocrino: reactividad, plasticidad y subjetividad (Santibáñez-H, 1986, pp. 4275). En la sintomatología están envueltos procesos reactivos de excitabilidad neuronal (Santibáñez-H, 1986, pp. 347-357), función básica de la actividad neuropsicológica cuya modificación "por la experiencia", es resultado del aprendizaje (Konorski, 1979), todo lo cual afecta la integración de los procesos subjetivos. De ahí que esta patología afecte tanto fenómenos funcionales viscerales, como el comportamiento y la subjetividad.

Sin lugar a dudas intervienen también otros factores como los condicionantes genéticos y las experiencias tempranas, que Korda estudió en perros (Korda, 1986) y gatos (Korda, 1978), Novakova (1986) en varias especies poniendo claramente de manifiesto la influencia de la experiencia temprana en el desarrollo del comportamiento, Novakova y Babinsky (1977) el rol de la experiencia en el comportamiento social de las ratas con sus crías y Novakova, Faltin, Flandera, Háhn y Koldovsky (1964), el efecto de la prematura separación de la madre sobre la memoria en ratas macho y los mismos autores (1962) sobre los efectos de la separación tardía en el aprendizaje en el estado adulto. 
La sociedad moderna industrial genera condiciones de vida en las cuales concurren los tres elementos señalados por Chananashwili. Los habitantes de los países industrializados están sometidos a grandes tensiones informacionales causadas por la intensa presión propagandística que opera sobre grandes masas de consumidores.

Restian (2001) al comentar la patología informacional pone de relieve que la explosión informacional que se ha generado en el proceso de transformación industrial caracterizada por un creciente incremento de la producción de bienes tecnológicos, incluidos los medios de comunicación, cuyo volumen ha alcanzado un nivel notable. Los volúmenes de los procesos informacionales sobrepasan los limites de la capacidad receptiva de quienes reciben estas informaciones. Se calcula que a escala mundial se publican anualmente 100 mil revistas científicas las cuales pueden incluir alrededor de un par de millones de contribuciones científicas.

Agréguese a esto que se publican $400 \mathrm{mil}$ periódicos, entre los cuales aproximadamente existen 800 de circulación diaria, entre todos publican cerca de 400 millones de ejemplares. Fuera de la información escrita existe un flujo informacional que es canalizado por aproximadamente mil millones de receptores de radio, sin contar los cientos de miles de televisores, además de mil millones de teléfonos incluidos 100 millones de teléfonos móviles, faxes, etc. Se calcula que existen 200 millones de PC, de los cuales, por lo menos el $30 \%$ están conectados a Internet. Todas esta cifras se incrementan día a día.

Es evidente que la explosión informacional modula negativamente la actividad diaria, incluida la profesional, pues interfiere considerablemente con el tiempo realmente libre. La información, verdadera o falsa, de tipo industrial, financiera y comercial se introduce silenciosamente en el mundo subjetivo de las personas. Esta información, lejos de "informar", opera compulsivamente sobre las decisiones periódicas impeliéndolas a adquirir productos que las más de las veces no concuerdan con las propiedades atribuidas en los avisos. Esta influencia imperativa, facilitada por el enorme tiempo empleado en atender a los programas de radio y televisión, así como las llamadas telefónicas, tiene indiscutiblemente una cierta eficacia. En una vida de 60 años se reciben alrededor de 50 millones de avisos de propaganda.

Se calcula que le $80 \%$ de la información contemporánea se establece por medios analógicos o digitales, siendo una gran proporción transmitida por Internet. Aproximadamente 100 millones de personas utiliza Internet para intercambiar mensajes "email", los cuales pueden alcanzar los $610 \mathrm{mil}$ millones al año.

Este impacto puede apreciarse mejor si se consideran algunos índices de la dinámica informacional. Se estima que el organismo recibe alrededor de 10 millones de bit/seg., de los cuales 1 millón son asimilados por el sistema neuroendocrino, codificados y luego integrados en la génesis de las reacciones del organismo dirigidas al medio externo, al medio interno y al medio subjetivo o intracerebral.

El organismo humano parece tener limites dentro de los cuales la información efectivamente favorece la adaptación. Flujos informacionales que quedan bajo el umbral de efectividad como también aquellos que los sobrepasan producen alteraciones relacionadas con los mecanismos del estrés: por ejemplo, aumento de corticoides, de catecolaminas, alteraciones cardiovasculares, etc.

La escasez de información produce síntomas de deprivación sensorial, manifestada por ansiedad, alteraciones del esquema corporal, alucinaciones. El exceso induce estrés, originando cansancio, ansiedad, irritación, alteraciones del sueño, palpitacio- 
nes, dolores precordiales y abdominales, cefaleas, temblores y otros.

El excesivo amor a los computadores tiene efectos patológicos serios como aislamiento social, falta de información para llevar a cabo la vida rutinaria, dificulta la realización necesaria para una vida medianamente normal.

Las sociedades industriales desarrolladas han alcanzado niveles exorbitantes de neurosis, de patologías psicosomáticas y emocionales que afectan a sus miembros, tanto en el aspecto físico como en el subjetivo. Miedo, angustia, inseguridad, etc., son los elementos básicos que generan la desadaptación contemporánea.

Ni discursos, ni conversaciones, ni prédica pueden contrarrestar el efecto patogénico de una actividad que, al parecer de los expertos en procesos económicos, mantiene el desarrollo y el progreso social.

\section{Referencias Bibliográficas}

Airapetyants, M.G. (1986) "From stress to Neurosis", in "Introduction to the physiopathology of Neurotic States", Edited by G. Santibáñez-H and M. Lindemann, VEB Georg Thieme Verlag, Leipzig, Alemania, págs. 134-139.

Airapetyants, M.G. and A.M. Vein (1982) "Neurosis in experiments and in clinic" (in Russian), Ed. Nanka, Moscú. Pp. 272.

Biondi, M. AND A. PiCARdi (1996) "Psychological Stress and Neuroendocrine functions in humans: The Last Two Decades of Research" Publicado por Dipartimento di Scienze Psichiatriche e Medicinina Psicologica, Roma, Italia

Chananashwili, M.M. (1984) "Informationsneurosen", en "Neurosen, Theorie und Expe- riment", edited by Chananashwili M.M. and Hecht, K., Akademie Verlag, Berlin, Alemania. Págs. 274-317.

Korda, P. (1979) "Locomotor Activity in Visually deprived kittens", Acta Neurobiológica Experimental, 38: 343-351.

KordA, P. (1986) "The Development of the Neurotic States and its Behavioral Model: Early Experience" in "Introduction to the physiopathology of Neurotic States", Edited by G. Santibáñez-H and M. Lindemann, VEB Georg Thieme Verlag, Leipzig, Alemania. Pp 357.

Natelson, B. (1983) "Stress, Predisposition and the Onset of Serious Disease: Implications About Psychosomatic Etiology", Neurosciences and Biobehavioral Disease 7: 511-527.

Nikolov, N.A. (1956) "Influence of the Higher Nervous Activity of Dogs" (in Russian) Fiziol. Zh. SSSR, 17: 925-930.

NiLKOlov, N.A. (1986) “General Adaptation Syndrome with Reference to the Problem of Individual Nerve Reactivity", in "Introduction to the physiopathology of Neurotic States", Edited by G. Santibáñez$\mathrm{H}$ and $\mathrm{M}$. Lindemann, VEB Georg Thieme Verlag, Leipzig, Alemania, págs.114-122.

Novakova, V. (1986) “Early experience and its Modelating Effects on the actual adaptations of the Organism", en "Introduction to the physiopathology of Neurotic States", Edited by G. Santibáñez$\mathrm{H}$ and $\mathrm{M}$. Lindemann, VEB Georg Thieme Verlag, Leipzig, Alemania. Pp 357.

Novakova, V., J. Faltin, V. Flandera, P. Háhn AND O. KolaOVSKY (1962) "Effect of Early and Late Weaning on Learning in adultosts", Nature 123: 280.

Novakova, V., O. Kolaovsky, J. Faltin, P. HáHn AND V. Flandera (1964) “The Effect 
of Premature Weaning and High Fat Diet on Relation of a Memory Trace in Male Rats", Physiol. Bohemoslov 12: 533-540.

Novakova, V. AND A. Babinsky (1977) “Role of Early Experience in Social Behaviour of Laboratory-Bred Female Rats", Behav. Proc. 2: 243-253.

Pavlov, I.P. (1934) “An Attempt at Physiological Interpretation of Obsessional Neurosis and Paranoia" The Journal of Mental Science 80: 187-197.

Restian, A. (2001) "Importanta Patologiei Informationale in Societatea Informationala" British Medical Journal (Editia in Limba Romána) Vol. 8, $N^{\circ}$ 9, en www.bmj.ro/numar/2001nr09/opinii1.html

SANTiBÁÑEZ-H, G. (1986) “Principal Functions of the Neuroendocrine System: The Functional Basis of the Integrative Activity of the Brain", en "Introduction to the physiopathology of Neurotic States", Edited by G. Santibáñez-H and M. Lindemann, VEB Georg Thieme Verlag, Leipzig, Alemania, págs. 43-75.

Santibáñez-H， G. (1986) “General Physiopathological Aspect of Neurotic States", en "Introduction to the physiopathology of Neurotic States", Edited by G. Santibáñez-H and M. Lindemann, VEB Georg Thieme Verlag, Leipzig, Alemania, págs. 237-259.

SAntibáÑez-H， G. (1986) "Integrative Functions of the Brain and Therapy of
Neurosis", en "Introduction to the physiopathology of Neurotic States", Edited by G. Santibáñez-H and M. Lindemann, VEB Georg Thieme Verlag, Leipzig, Alemania, págs. 347-357.

Selye, H. (1950) "The Physiology and Pathology of Exposure to Stress", Acta Inc. Medical Publishers, Montreal, Canada. Pp 822.

Selye, H. (1956) "What is Stress?", Metabolism 5: 525-530.

Sudakov, K.V. (1980) “The Dynamics of Cardiovascular Disorders in Rabbits Under Acute Experimental Emotional Stress" (in Russian), Bull. Exp. Biol. i. Med. 89: 665-664.

SuDAKOV, K.V. (1986) “Emotional Stress", en "Introduction to the physiopathology of Neurotic States", Edited by G. Santibáñez$\mathrm{H}$ and $\mathrm{M}$. Lindemann, VEB Georg Thieme Verlag, Leipzig, Alemania, págs. 123-133.

Telchanov, L. y N.A. Nikolov (1963) "Reactividad Normal y Patológica" (en Búlgaro), "Khristo G. Danov", Ploudiv.

Periodo de límite de recepción de artículos: 30 de Marzo 2002.

Fecha de aceptación artículos: 30 de octubre 2002. 